\title{
Lens-Less Foucault Imaging (LLFI) Method for Observing Magnetic Domain Walls
}

\author{
Yoshifumi Taniguchi ${ }^{1}$, Hiroaki Matsumoto ${ }^{2}$ and Ken Harada ${ }^{3}$
}

1. Advanced Microscope System Design Dept., Hitachi High-Technologies Corp., Ichige, Hitachinaka, Ibaraki 321-8504, JAPAN

2. Application Development Dept., Hitachi High-Technologies Corp., Ichige, Hitachinaka, Ibaraki 3120033, JAPAN

3. Central Research Laboratory, Hitachi Ltd., Hatoyama, Saitama 350-0395, JAPAN

The conventional Foucault imaging method, an optical mode of Lorentz microscopy, can visualize magnetic domains under infocus condition. Imaging of the Foucault mode, however, requires a magnetic-field shielding lens and an off-centered objective aperture. As microscopes have to be customized for observing magnetic domain structures, the Foucault mode has not been used in practice. Recently, however, a novel Foucault imaging method, named lens-less Foucault imaging (LLFI) [1], was developed for conventional transmission electron microscopes without any special equipment for Lorentz microscopy.

Figure 1(a) shows the optical system of LLFI in a 300-kV field-emission TEM (HF-3300 made by Hitachi High-Technologies Corp). The objective lens was switched off and the electron beam was converged with a condenser lens to the crossover on the selected area aperture plane. The selected area aperture was used as an objective aperture to select the deflected beam for the Foucault mode, and the focal length of the magnifying lens was changed in order to observe the specimen images and diffractions. The irradiated area on the specimen was set by selecting an appropriate diameter for the condenser aperture. Figure 1(b) is an example of small-angle electron diffraction of a $90^{\circ}$ ferromagnetic domain structure of $\mathrm{La}_{0.75} \mathrm{Sr}_{0.25} \mathrm{MnO}_{3}$ (LSMO). The observation was done with a camera length of $150 \mathrm{~m}$.

Figure 2 shows Foucault images from a single dispersed deflection spot of Fig. 1(b) made with a selected area aperture of $5 \mu \mathrm{m}$ diameter. The magnetization distribution among the $90^{\circ}$ domains is directly visualized.

Figure 3 shows Foucault images of the magnetic domain walls in the same area as Figure 2. Figure 3(a) shows $180^{\circ}$ domain walls imaged with two streaks including the optical axis. Figures 3 (b) and (c) are $90^{\circ}$ domain walls imaged with each streak between two spots with an aperture $3 \mu \mathrm{m}$ in diameter. Figure $3(\mathrm{~d})$ shows the whole domain walls in the same area imaged by using an aperture $5 \mu \mathrm{m}$ in diameter.

LLFI method is advantageous for observing not only magnetic domains and domain walls but also small-angle diffractions and deflections less than $2 \times 10^{-5}$ rad. The LLFI method will open the way to developing new applications in Lorentz microscopy.

\section{References:}

[1] Y. Taniguchi et al., Appl. Phys. Lett., 101, 093101 (2012).

[2] The LSMO specimens were supplied by Prof. S. Mori of Osaka Prefecture University. 
(a)
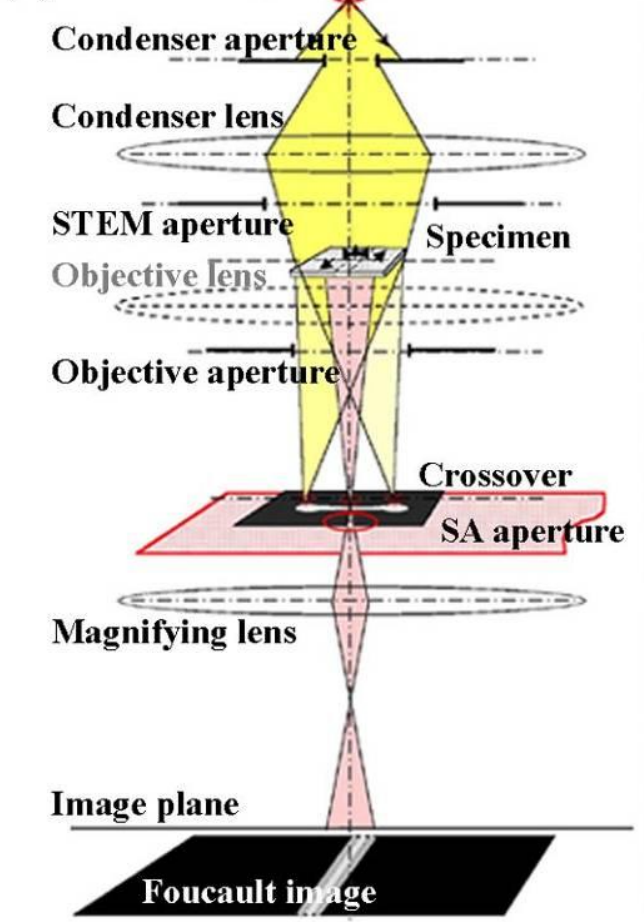

(b)

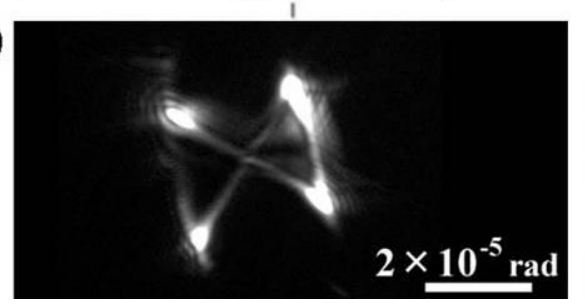

Figure 1
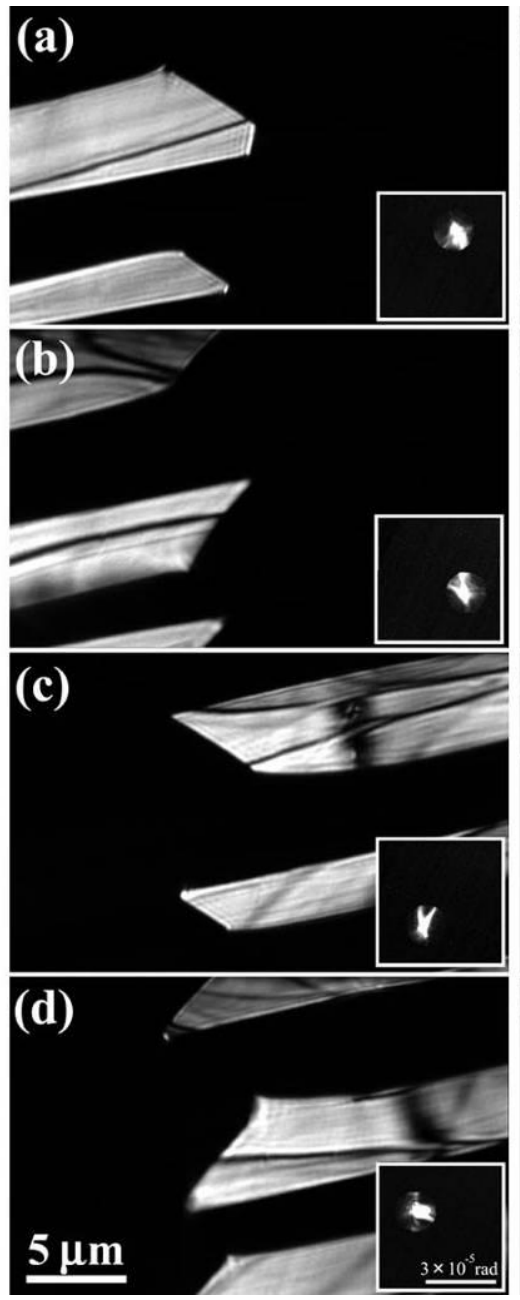

Figure 2
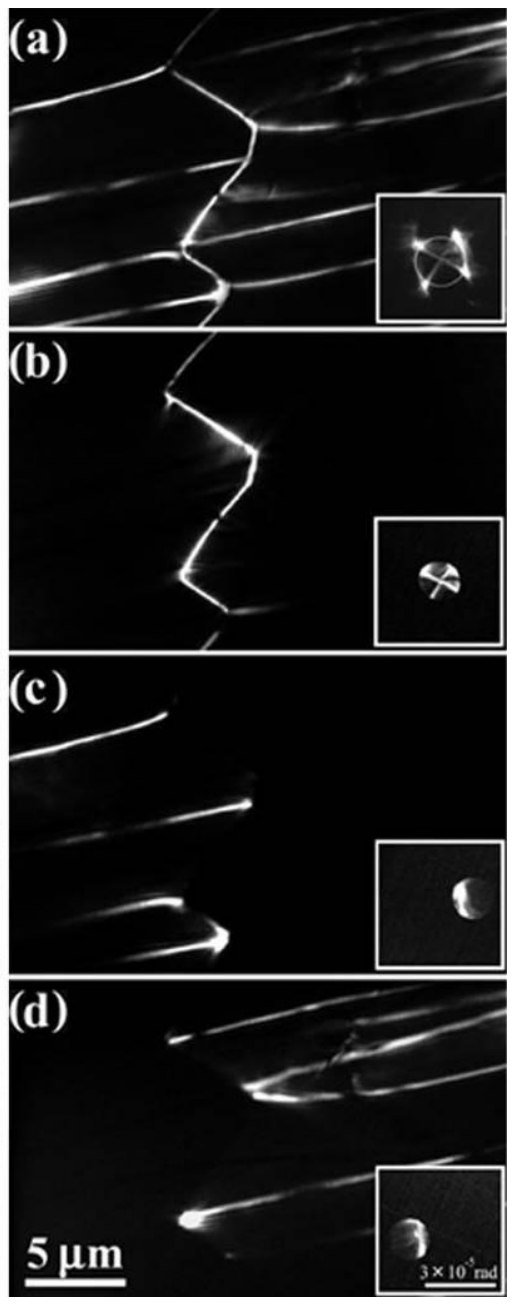

Figure 3

Figure 1. (a) Optical system for LLFI method, (b) small-angle electron diffraction from $907180^{\circ}$ domain structure of LSMO.

Figure 2. Foucault images of $90^{\circ}$ domains from each single deflection spot.

Figure 3. Foucault images of domain walls, (a) $180^{\circ}$ domain walls, (b) $90^{\circ}$ domain walls, (c) $90^{\circ}$ domain walls, and (d) $90^{\circ} / 180^{\circ}$ domain walls. 